\title{
Ovaryan ven reflüsünün değerlendirilmesinde arteriyel ve erken portal venöz faz BT görüntülerinin karşılaştırılması
}

\author{
Kaan Esen"1, Sermin Tok Umay', Yüksel Balcı1 ${ }^{1}$ Anıl Özgür1', \\ Feramuz Demir Apaydın' \\ Mersin Üniversitesi Tıp Fakültesi Radyoloji Ana Bilim Dalı, Mersin
}

Öz

Amaç: Dinamik bilgisayarlı tomografi tetkikinde ovaryan venlerde izlenebilen reflü açısından arteriyel ve erken portal venöz faz görüntüleri karşılaștırılmıştır. Yöntem: Ocak 2010-Mart 2016 tarihleri arasında kliniğimizde yapılan dinamik bilgisayarlı tomografi tetkikleri retrospektif olarak incelendi ve 125 hasta çalışmaya dahil edildi. Kontrast madde verilmeye başlanmasından sonra 25. saniyede (arteriyel faz) ve 60. saniyede (erken portal venöz faz) elde edilen görüntüler incelendi. Ovaryan venlerdeki reflü varlığı değerlendirildi. Sol ve sağ ovaryan venlerin çapları ölçüldü. Bulgular: Yüz yirmi beş hastanın 29'unda (\%23,2), hem arteriyel hem de erken portal venöz fazda sol ovaryan vende reflü saptandı. Otuz üç $(\% 26,4)$ hastada sadece erken portal venöz fazda sol ovaryan ven reflüsü tespit edildi. Kırk dokuz $(\% 39,2)$ hastada erken portal venöz fazda sağ ovaryan ven reflüsü gözlendi. Sol ovaryan ven çapı ortanca değerlerinin; sol ovaryan vende sadece erken portal venöz fazda reflüsü olanlarda reflü olmayanlara göre daha yüksek $(p=0,016)$, her iki fazda da reflüsü olanlarda reflü olmayanlara göre çok daha yüksek $(p<0,001)$ ve yine her iki fazda reflüsü olanlarda sadece erken portal venöz fazda reflü olanlara göre yüksek olduğu $(p<0,001)$ gözlendi. Sonuç: Sol ovaryan vende erken portal venöz fazda reflü saptanan bazı hastalarda arteriyel fazda ovaryan vende reflü saptanmaması, arteriyel fazın over venlerindeki reflünün değerlendirilmesi için yeterli olmadığını göstermektedir. Ancak özellikle sol ovaryan ven çapı geniş olan hastalarda arteriyel fazda da reflünün görülmesi bu hastalardaki reflünün şiddeti ile ilişkili olabilir. Bu nedenle, ovaryan venlerde reflünün görülmeye başladığı zaman, ovaryan ven çapı ve reflünün şiddeti arasındaki ilişkinin değerlendirileceği prospektif çalışmalarla BT'nin pelvik konjesyon tanısındaki etkinliği artacaktır.

Anahtar kelimeler: Ovaryan ven, pelvik konjesyon, reflü

\section{Comparison of arterial and early portal venous phase CT images in the evaluation of ovarian vein reflux}

\begin{abstract}
Aim: On dynamic CT scan, arterial and early portal venous phase images were compared in terms of reflux in ovarian veins. Methods: Dynamic CT scans performed in our clinic between January 2010 and March 2016 were reviewed retrospectively and 125 patients were included in the study. After the initiation of contrast medium administration, images were obtained with a scan delay of 25 (arterial phase) and 60 (early portal venous phase) seconds were evaluated.
\end{abstract}

Başvuru tarihi: 05.10.2017

Kabul tarihi: 05.12.2017

Sorumlu Yazar: Yrd. Doç. Dr. Kaan Esen: Mersin Üniv. Tıp Fak. Hastanesi Radyoloji Bölümü 34. Cadde Yenişehir/Mersin, Tel: 324 2410000-532 4256554

e posta: kaanessen@yahoo.com 
The presence of reflux in ovarian veins was evaluated. Diameters of left and right ovarian veins were measured. Results: Left ovarian vein reflux was detected in 29 (23.2\%) of 125 patients in both arterial and early portal venous phase. Thirty three (26.4\%) patients had left ovarian vein reflux only in early portal venous phase. Forty nine (39.2\%) patients had right ovarian vein reflux in early portal venous phase. The median values of the left ovarian vein diameter were significantly higher in the patients with left ovarian vein reflux only in early portal venous phase than those without reflux ( $p=0.016)$. The median values of the left ovarian vein diameter were also significantly higher in the patients with reflux in both phases than those without reflux $(p<0.001)$ and with reflux only in early portal venous phase $(p<0.001)$. Conclusion: The absence of arterial phase reflux in some patients who have reflux in early portal venous phase suggests that the arterial phase is not sufficient to assess reflux in the left ovarian vein. However, detection of reflux in arterial phase, especially in the patients with large left ovarian vein, may be related with the severity of reflux in these patients. Therefore, the evaluation of the relationship between the beginning phase of reflux in the left ovarian vein, ovarian vein diameter and reflux severity with prospective studies will increase the efficacy of CT in the diagnosis of pelvic congestion syndrome.

Keywords: Ovarian vein, pelvic congestion, reflux

\section{Giriş}

Pelvik konjesyon sendromu organik bir neden olmaksızın kronik pelvik ağrı ile karakterize olup genellikle multipar kadınlarda görülür. ${ }^{1,2}$ Ovaryan vende izlenen reflü ve dilatasyonun hastalığın gelişiminde önemli bir etyolojik faktör olduğuna inanılmaktadır. ${ }^{1}$ Bununla birlikte, yapılan çalışmalarda asemptomatik bireylerde de ovaryan vende reflünün bulunabileceği gösterilmiştir..$^{1,2,3}$ Hastalığın tanısında faydalı bir görüntüleme yöntemi olan kontrastlı bilgisayarlı tomografi (BT) ile ovaryan venlerde reflü gösterilebilir. Renal venlerin opasifikasyonu ile birlikte ovaryan vende görülen opaklaşma retrograd venöz akıma işaret eder. ${ }^{1}$ Ancak incelemenin hangi fazda gerçekleștirilmesi gerektiği konusu tartışmalıdır. Literatürde farklı özellikteki BT cihazları ile yapılan çalışmalarda, intravenöz yolla kontrast madde enjeksiyonu başladıktan sonra 25. saniye 95. saniye arasında ovaryan venlerdeki reflünün bildirilmiştir. ${ }^{1,2,3}$ değerlendirilebileceği

Bu çalışmada, 64 kesitli BT cihazı ile gerçekleştirilmiş olan dinamik çok kesitli BT (ÇKBT) incelemelerinde, ovaryan vende reflü varlığı açısından, arteriyel faz (25. saniye) ve erken portal venöz fazda ( 60 . saniye) elde edilen görüntüler karşılaştırılmıştır.

\section{Gereç ve Yöntem}

Yapılan başvuruda, Mersin Üniversitesi Klinik Araştırmalar Etik Kurulu tarafından araştırmanın gerçekleştirilmesinde etik olarak sakınca yoktur kararl (14.04.2016, 2016/93) alındıktan sonra çalışma başlatıldı. Ocak 2010-Mart 2016 tarihleri arasında kliniğimizde yapılan dinamik BT incelemeleri retrospektif olarak incelendi. Dinamik incelemelerin çoğunluğu karaciğer patolojilerine yönelik olarak yapılmıştı. Ovaryan ven akımını etkileyebilecek karın içi kitlesi olan hastalar (retroperitoneal kitle, paraaortik lenfadenopati, vb), histerektomi, ooferektomi, nefrektomi öyküsü olan hastalar ve portal hipertansiyonu olan hastalar çalışma dışı bırakıldı. ${ }^{3}$ Ayrıca hareket artefaktları gibi nedenlerle görüntü kalitesi yetersiz olan tetkikler ve uygun kontrast fazında gerçekleştirilemeyen tetkikler çalışma dışı bırakıldı. Toplam 125 kadın hasta çalışma grubu olarak kabul edildi.

Dinamik BT tetkikleri 64 kesitli cihaz (Toshiba Aquilion 64, Toshiba Medical Systems, Tokyo, Japonya) kullanılarak gerçekleştirildi. Arteriyel ve erken portal faz görüntüleri her fazda nefes tutmalı olarak, 100-120 ml noniyonik iyotlu kontrast maddenin $3-4 \mathrm{ml} / \mathrm{s}$ hizla intravenöz olarak verilmeye başlanmasından 25 saniye ve 60 saniye sonra elde edildi. Tarama alanı 
diyafram kubbesi düzeyinden iliak kemiklerin üst kısmına kadardı.

Görüntüler deneyimli iki radyolog tarafından görüș birliğiyle değerlendirildi. Arteriyel ve erken portal venöz fazlarda ovaryan venlerde izlenen opaklaşma reflü olarak kabul edildi. Reflü derecesi gözardı edilerek sadece reflü varlığı değerlendirildi. Sol ve sağ ovaryan venlerin çapları transvers görüntülerde ölçüldü ve kaydedildi. Her damar için iki ölçüm yapıldı ve analiz için ortalama değer kullanıldı. Değerlendirmede koronal ve MIP (maksimum intensite projeksiyon) görüntülerden de faydalanıldı.

Sürekli ölçümlere ait normallik kontrolleri Shapiro Wilk testi ile test edildi. Sağ ve sol ovaryan vende reflü varlığı ile sağ ve sol ovaryan ven çapları arasındaki ilişki Mann Whitney U testi ve Kruskall Wallis testleri ile test edildi. İkili karşılaştırmalar için Mann Whitney U testinden yararlanıldı. Tanımlayıcı istatistikler olarak ortanca, \%25-75 çeyreklikler kullanıldı. Ayrıca, sağ ve sol ovaryan veninde reflü olan hastaların yaș ortalamaları Student t testi ile test edildi. Tanımlayıcı istatistikler olarak ortalama ve standart sapma değerleri verildi. İstatistik anlamlılık için $\mathrm{p}<0.05$ alındı.

\section{Bulgular}

Çalışmaya dahil olan 125 hastanın yaş ortalaması $51.3 \pm 11.7$ (26-83) olarak tespit edildi.

Hem arteriyel hem de erken portal venöz fazda 125 kadının 29'unda (\%23.2) sol ovaryan vende reflü saptandı (Resim 1). Sadece erken portal venöz fazda sol ovaryan ven reflüsü 33 (\%26.4) hastada tespit edildi (Resim 2). Altmış üç (\%50.4) hastanın sol ovaryan veninde reflü izlenmedi. Kırk dokuz (\%39.2) hastanın sağ ovaryan veninde erken portal venöz fazda reflü gözlendi.

Sağ ve sol ovaryan venlerin ortalama çap değerleri Tablo 1'de verilmiştir.

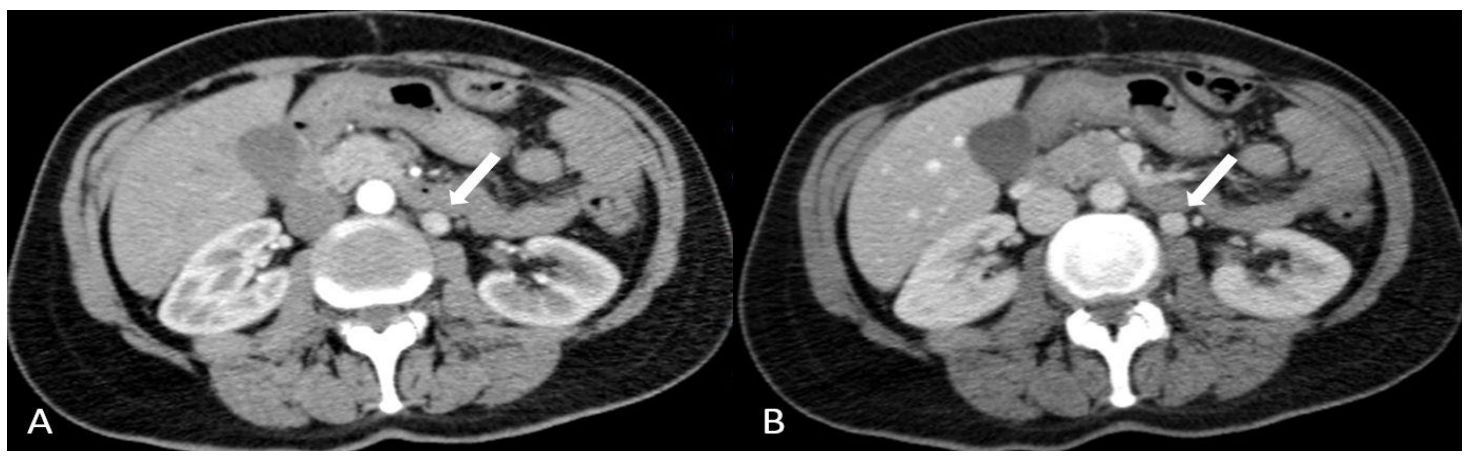

Resim 1. Arteriyel faz (A) ve erken portal venöz faz (B) aksiyel BT görüntülerinde sol ovaryan venin geniş olduğu ve her iki fazda da reflünün bulunduğu izlenmekte (oklar).

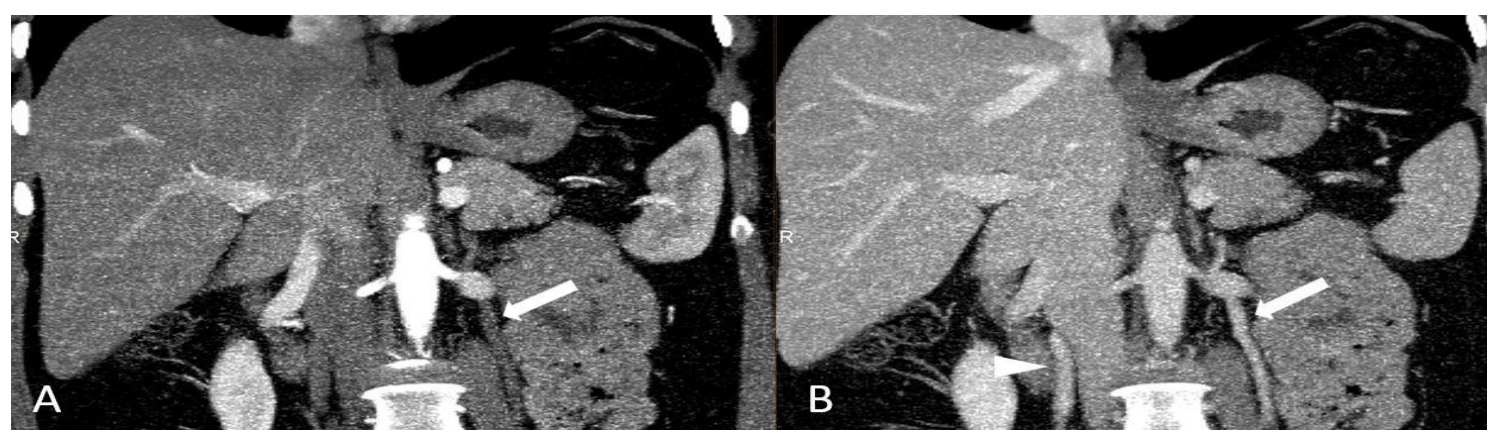

Resim 2. Arteriyel faz (A) koronal maksimum intensite projeksiyon BT görüntülerinde sol ovaryan vende reflü izlenmezken (ok), erken portal venöz faz (B) görüntülerinde sol ovaryan vende reflü dikkati çekmekte (ok). Erken portal venöz fazda sağ ovaryan vende de reflü mevcut (ok başı). 
Tablo 2. Hasta yaşı ortalama değerlerinin ve ovaryan ven çapı ortanca değerlerinin ovaryan vendeki reflü varlığına göre dağılımı.

\begin{tabular}{|c|c|c|c|c|c|c|}
\hline & Ovaryan Vende Reflü & Hasta & & Ovar & n Ven Çapı ( & m) \\
\hline & & Ort \pm SD & $\mathrm{P}$ & Min-Maks & $\begin{array}{c}\text { Ortanca } \\
{[\% 25-75]}\end{array}$ & $\mathrm{P}$ \\
\hline & Yok $(n=63)$ & $53.4 \pm 12.0$ & 0.047 & $1.9-9$ & 3.8 & $<0.001$ \\
\hline 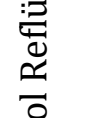 & $\begin{array}{l}\text { Erken portal fazda var } \\
(\mathrm{n}=33)\end{array}$ & $51.1 \pm 11.6$ & & $2.3-7.6$ & $\begin{array}{c}{[3.0-4.6]} \\
4.3^{*} \\
{[3.85-5.25]}\end{array}$ & \\
\hline s & Her iki fazda var $(n=29)$ & $47.0 \pm 10.1^{*}$ & & $4.1-11$ & $\begin{array}{c}7.5^{*} \cdot \dagger \\
{[5.40-8.95]}\end{array}$ & \\
\hline & Yok $(n=76)$ & $52.7 \pm 11.9$ & 0.096 & $0-9.2$ & $\begin{array}{c}4 \\
{[3-468}\end{array}$ & 0.004 \\
\hline$\tilde{\infty} \approx$ & $\begin{array}{l}\text { Erken portal fazda var } \\
(\mathrm{n}=49)\end{array}$ & $49.2 \pm 11.1$ & & $2.4-9.5$ & $\begin{array}{c}4.7^{*} \\
{[3.75-5.85]}\end{array}$ & \\
\hline
\end{tabular}

*: Reflü olmayan grup ile farklılıkları; $\uparrow$ : Erken portal fazda reflü olan grup ile farklılıkları göstermektedir.

Ovaryan venlerdeki reflünün araştırıldığı ve helikal BT ile yapılan ilk çalışmalarda ise reflü varlığı arteriyel fazda değerlendirilmiș olup kontrast injeksiyonunun başlamasından sonra 20-30 saniyelik bir gecikmeyle tarama başlatılmıștır.1,7 Bununla birlikte, ÇKBT ile yapılan bir çalışmada kontrast madde enjeksiyonu başladıktan sonra 50-80. saniyelerde tarama yapılarak ovaryan venlerin yanısıra parauterin ve uterin venlerdeki reflü de değerlendirilmiştir. ${ }^{2}$ Koç ve arkadaşlarl $^{3}$ ise ÇKBT ile portal fazda alınan görüntülerde sol renal ven varyasyonlarının pelvik varisler ve sol ovaryan vendeki reflü ile ilişkisini araştırmıştır. Eski nesil BT cihazları ile karşılaştırıldığında günümüzde kullanılan ÇKBT cihazları ile yapılan taramalar daha kısa sürelerde tamamlanabilmektedir. $\mathrm{Bu}$ nedenle literatürde ovaryan venlere yönelik yapılan çalışmalarla karşılaştırdığımızda çalışmamızda arteriyel faz görüntülerin daha erken sürelerde elde edildiğini düşünüyoruz.

Rutin olarak yapılan dinamik üst batın BT tetkiklerinde ovaryan venlerdeki genişleme ve reflü genellikle dikkate alınmamakta ve raporlanmamaktadır. Ayrıca uterus ve overler tetkik kapsaminda olmadığından parauterin venlerde genişleme olsa bile değerlendirilememektedir. Ancak üst batın BT tetkiklerinde saptanan ovaryan venlerdeki reflü ve genişleme olası bir pelvik konjesyon sendromu yönünden önemli bir ipucu olabilir. Bununla birlikte yapılan çalıșmalarda pelvik konjesyon sendromu açısından asemptomatik bireylerin \%3847'sinde ovaryan vende reflünün görülebileceği bildirilmiștir.1,2,8 Ayrıca doğum yapmış asemptomatik kadınlarda reflü izlenen sol ovaryan ven çapının reflü bulunmayanlara göre daha geniş olduğu rapor edilmiștir. ${ }^{2}$ Normal bireylerde ovaryan ven çapı için kabul edilen üst limit yaklaşık 5mm'dir. ${ }^{3}$ Ovaryan ven çapının $>7-8 \mathrm{~mm}$ olması pelvik konjesyon sendromu için destekleyici bir bulgu olarak kabul edilmekle birlikte yaygın olarak kabul görmüş bir değer bulunmamaktadır. ${ }^{4,9} \mathrm{Bu}$ nedenle tek başına ovaryan vende genişleme veya reflünün tespit edilmesi pelvik konjesyon sendromu tanısı için yeterli olmayıp hastaların klinik olarak da değerlendirilmesi gerekmektedir.

Ovaryan venlerde reflünün değerlendirilmesinde ÇKBT etkili bir görüntüleme yöntemidir. Çok kesitli bilgisayarlı tomografi cihazları ile yapılan dinamik incelemelerde bazı hastalarda sol ovaryan vende erken portal venöz fazda reflü saptanabilir. Bu hastalarda arteriyel fazda reflü saptanmaması ovaryan venlerdeki reflünün ortaya konulması için arteriyel fazın erken bir faz olabileceğini düşündürebilir. Ancak özellikle arteriyel fazda reflünün görülmeye başlandığı hastalarda sol ovaryan venin geniş olması reflünün daha şiddetli olabileceğini düşündürmektedir. $\mathrm{Bu}$ durumun pelvik konjesyon sendromu ile ilişkisinin araștırılması gerektiğini düşünmekteyiz. 
Çalıșmamızın bazı kısıtlılıkları bulunmakta olup bunlardan en önemlisi retrospektif olarak yapılmış olmasıdır. Hastaların pelvik konjesyon sendromu açısından klinik olarak değerlendirilememesi, gebelik ve doğum sayılarına ulaşılamaması diğer önemli kısitlılıklardır.

\section{Sonuç}

Sol ovaryan vendeki çap artışı ile birlikte reflünün arteriyel fazdan itibaren görülebiliyor olması arasında ortaya koyduğumuz istatistiksel ilişki, bu konuda yeni çalışmalara ihtiyaç duyulduğunu göstermektedir. Pelvik konjesyon sendromu açısından semptomatik ve asemptomatik kişilerde reflünün görülmeye başladığı zaman, ovaryan ven çapı ve reflünün şiddeti arasındaki ilişki prospektif çalışmalarla değerlendirilmelidir.

\section{Kaynaklar}

1. Rozenblit AM, Ricci ZJ, Tuvia J, Amis ES Jr. Incompetent and dilated ovarian veins: $A$ common CT finding in asymptomatic parous women. AJR Am J Roentgenol 2001;176(1):119-122.

2. Hiromura T, Nishioka T, Nishioka S, Ikeda $\mathrm{H}$, Tomita K. Reflux in the left ovarian vein: Analysis of MDCT findings in asymptomatic women. $A J R \quad A m \quad J$ Roentgenol 2004;183(5):1411-1415.
3. Koc Z, Ulusan S, Oguzkurt L. Association of left renal vein variations and pelvic varices in abdominal MDCT. Eur Radiol 2007;17(5):1267-1274.

4. Cura M, Cura A. What is the significance of ovarian vein reflux detected by computed tomography in patients with pelvic pain? Clin Imaging 2009;33(4):306-310.

5. Sheth S, Fishman EK. Multi-detector row CT of the kidneys and urinary tract: techniques and applications in the diagnosis of benign diseases. Radiographics 2004;24(2):e20.

6. Tsuge $Y$, Kanematsu M, Goshima S, at al. Optimal scan delays for multiphasic renal multidetector row computed tomography performed with fixed injection duration of contrast medium. J Comput Assist Tomogr 2009;33(1):101-105.

7. Desimpelaere JH, Seynaeve PC, Hagers YM, Appel BJ, Mortelmans LL. Pelvic congestion syndrome: Demonstration and diagnosis by helical CT. Abdom Imaging 1999;24(1):100-102.

8. Nascimento AB, Mitchell DG, Holland G. Ovarian veins: Magnetic resonance imaging findings in an asymptomatic population. J Magn Reson Imaging 2002;15(5):551-556.

9. Coakley FV, Varghese SL, Hricak H. CT and MRI of pelvic varices in women. J Comput Assist Tomogr 1999;23:429-443 